\title{
Detection of phase transition in polyethylene glycol using a multimodal micromechanical acoustic resonator
}

Cite as: Appl. Phys. Lett. 110, 134101 (2017); https://doi.org/10.1063/1.4979086

Submitted: 22 November 2016 . Accepted: 09 March 2017 . Published Online: 27 March 2017

A. Prasad (D), and A. A. Seshia (D)
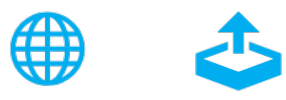

View Online

Export Citation

\section{ARTICLES YOU MAY BE INTERESTED IN}

Suppression of conductivity deterioration of copper thin films by coating with atomic-layer materials

Applied Physics Letters 110, 131601 (2017); https://doi.org/10.1063/1.4979038

Neutralization of an epitaxial graphene grown on a SiC(0001) by means of palladium intercalation

Applied Physics Letters 110, 131602 (2017); https://doi.org/10.1063/1.4979083

Frequency latching in nonlinear micromechanical resonators

Applied Physics Letters 110, 143506 (2017); https://doi.org/10.1063/1.4979829

Lock-in Amplifiers Find out more today

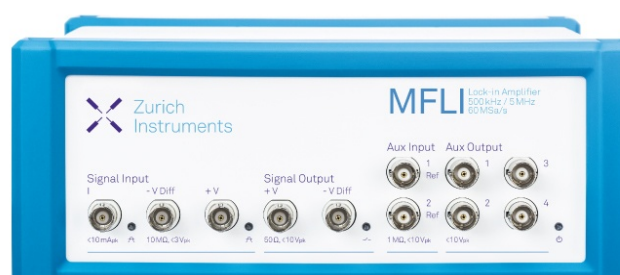

Zurich Instruments 


\title{
Detection of phase transition in polyethylene glycol using a multimodal micromechanical acoustic resonator
}

\author{
A. Prasad ${ }^{1,2, a)}$ and A. A. Seshia ${ }^{1}$ \\ ${ }^{1}$ Nanoscience Centre, Department of Engineering, University of Cambridge, Cambridge CB30FF, \\ United Kingdom \\ ${ }^{2}$ Institute for Life Sciences, Department of Electronics and Computer Science, University of Southampton, \\ Southampton SO171BJ, United Kingdom
}

(Received 22 November 2016; accepted 9 March 2017; published online 27 March 2017)

\begin{abstract}
A piezoelectrically transduced micromechanical beam acoustic resonator is utilized to detect solid to liquid phase transitions in sub-nanoliter volume of Polyethylene Glycol (PEG) 1000. A lower frequency flexural mode and a higher frequency length-extensional mode are simultaneously monitored for changes in the device response. The phase transition of the PEG drop perturbs the acoustic loading of the resonator, bringing about characteristic and repeatable changes in the frequency response. The phase transitions in the drop are verified optically, and the resonant parameters are compared with the control case when the resonator is pristine without the presence of any PEG drop. Published by AIP Publishing. [http://dx.doi.org/10.1063/1.4979086]
\end{abstract}

Micro/nanomechanical resonators demonstrate a modulation in their frequency response due to attachment/detachment of analyte, ${ }^{1}$ interaction with the surrounding fluid, ${ }^{2}$ and changes in the ambient conditions. ${ }^{3,4}$ The resonant frequency modulation is used as the primary readout in gravimetric sensing applications where the resonant frequency is correlated to effective mass $m$ and effective stiffness $k$ of an acoustic resonator by the following expression:

$$
\delta f=\frac{1}{2}\left(\frac{\delta k}{k}-\frac{\delta m}{m}\right) f
$$

where $\delta k(\ll k)$ and $\delta m(\ll m)$ are perturbations in effective stiffness and mass of the acoustic resonator being transduced at a resonant frequency $f$, while $\delta f$ is the resulting change in the resonant frequency. Dissipation $\zeta$ or quality factor $Q$ $(=1 / 2 \zeta)$ is another sensor parameter which is often used to understand the processes responsible for causing changes in the resonator frequency response. ${ }^{5}$ While various topologies of micro-/nanoscale resonators have been explored for different applications, ${ }^{6}$ bulk-acoustic ${ }^{2}$ and flexural ${ }^{7}$ wave resonators are particularly interesting for sensing applications where the resonators are expected to be highly responsive to the ambient viscoelastic changes. Different transduction techniques (capacitive, ${ }^{8}$ piezoresistive, ${ }^{9}$ thermal, ${ }^{10}$ electromagnetic, ${ }^{7}$ and piezoelectric ${ }^{11}$ ) have been employed for actuation and sensing of resonant modes in such devices. In this letter, piezoelectric transduction was preferred due to the ease of implementation (improved electromechanical coupling relative to the other schemes) and the possibility of exciting multiple resonant modes, both bulk and flexural, simultaneously.

The phase and glass transition studies of materials are important in lyophilization or freeze-drying, ${ }^{12}$ characterization of polymers, ${ }^{13}$ inkjet printing, ${ }^{14}$ and coagulation studies. ${ }^{15}$ Several techniques (differential scanning calorimetry, ${ }^{16}$ thermo-gravimetric analysis, ${ }^{17}$ electrical-thermal analysis, ${ }^{18}$

\footnotetext{
${ }^{\text {a) }}$ Author to whom correspondence should be addressed. Electronic mail: abhinav.prsd@gmail.com
}

and microscopy ${ }^{19}$ ) are conventionally used for determining the dynamic transition behavior of the tested samples. However, when the samples are relatively small (less than a milligram), these techniques prove to be challenging and inefficient. Minimum sample mass which could be accurately tested with these conventional tools is often several milligrams or more, and thus require adaptations, ${ }^{20}$ which can address small mass without compromising on the accuracy. Since, both phase and glass transition involves significant changes in the mechanical properties of the material (elastic moduli, viscosity, and density), utilizing micromechanical acoustic resonators which are quite sensitive to these parameters could provide an interesting tool for such studies. Indeed, microcantilevers have been previously used to detect transitions among different rotator phases in nanogram samples of paraffin wax, ${ }^{21}$ and in a separate study, flexural plate wave resonators were used to characterize glass transition in different polymeric glues. ${ }^{22}$ In both the cases, resonators were uniformly coated with the samples and exposed to temperature variations. In the present study, instead of a uniform layer, phase transition (solid to liquid and vice versa) in a microdroplet of a waxy crystalline polymer, polyethyelene glycol (PEG) 1000 , is studied by observing the changes in the resonant parameters of two distinct piezoelectrically transduced resonant modes (flexural and bulk) of a beam resonator. Both the resonant modes demonstrated large negative resonant frequency shifts during phase transition (which was verified optically) in a PEG deposit of a mass of $182 \mathrm{ng}$ and were used to establish the phase transition temperature window of $309 \mathrm{~K}-318 \mathrm{~K}$. Hence, the proposed technique paves the way for studying the phase transitions in ultralow mass samples, which is currently challenging using the conventional tools.

Beam resonators are fabricated through multi-mask industrial PiezoMUMPs by MEMSCAP (supplementary material), where thin layers of piezoelectric film (AIN) and electrode (Al) are deposited and patterned over underlying device Si layer. After subsequent patterning of bonding pads, resonant structures are released though bulk micromachining. 
Beam resonators used in the current study are $1000 \mu \mathrm{m}$ in length, $250 \mu \mathrm{m}$ in width with a total thickness of about $11.5 \mu \mathrm{m}$. Resonators are suspended from the middle points of the longer edges through a couple of tethers (Fig. 1(a)). Si chips with the microfabricated resonators are then glued to the ceramic chip carrier (LCC44 Spectrum Semiconductor) and wire bonded using a wedge wire bonder. The resonators are kept inside a thermally controlled vacuum 4-probe station (Lakeshore RF probe station) where a stable pressure of $5 \times 10^{-5}$ Bar is reached before commencing experiments. A detailed discussion on the experimental set-up is provided in the supplementary material. A two port open-loop scheme, which involves using an $\mathrm{Al}$ electrode pair for sensing and actuation while grounding the $\mathrm{Si}$ device layer, is employed to perform electrical characterization. The transmission parameters of the resonators are recorded using a Network Analyser (Agilent 4396B). Before the PEG droplet experiment, a control experiment is performed on a similar but a pristine resonator without any PEG deposit. The PEG droplet is deposited onto the bottom surface of a fresh test resonator by initially melting a solid mass of PEG and then using one of the probes of the vacuum probe station to first dip into the liquefied mass and then carefully bringing the probe in close proximity to the resonator's surface such that the hanging drop wicks away towards the intended location. By using ImageJ and approximating the deposit with a half-ellipsoid, the volume of the drop is estimated to be around $167 \mathrm{pl}$ with a mass of $182 \mathrm{ng}$. Multiple cycles of heating and cooling are performed to remove excess absorbed water and other volatile species. Temperature sweeps are limited to $320 \mathrm{~K}$ as post phase transition, the kinematic viscosity of PEG changes non-linearly ${ }^{23}$ leading to enhanced wetting of the resonator surface which affects the overall contact area of the drop. During the experiments, approximately $5 \mathrm{~min}$ is allowed for ramping up and cooling down before recording each set of data. Phase transition is optically verified by a vertically mounted digital microscope (Fig 1(b)).

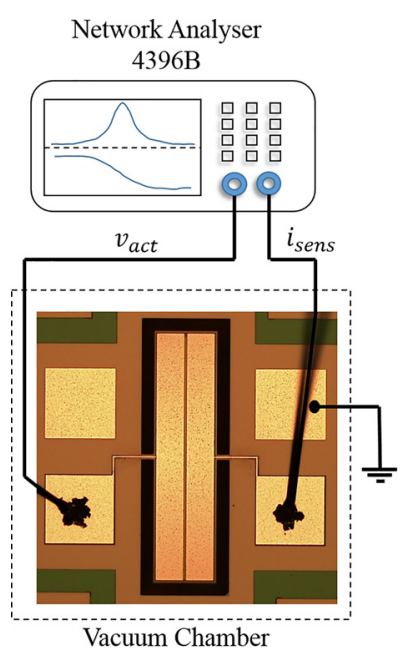

(a)

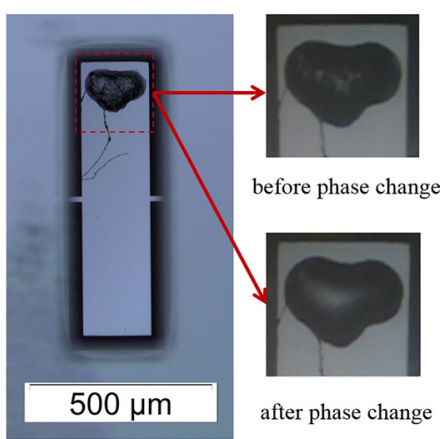

(b)
FIG. 1. (a) Circuit schematic of a beam resonator connected in a two port open loop scheme and (b) optical micrograph of the beam resonator (bottom surface) illustrating the location of polyethylene glycol 1000 drop before and after phase change (right).
The beam resonator is excited at a lower frequency ( $\sim 245 \mathrm{kHz}$ ) flexural mode M1 (inset Fig. 2(a)) and a higher frequency $(\sim 4.12 \mathrm{MHz})$ characteristic length-extensional mode M2 (inset Fig. 2(b)). The PEG droplet is located such that it lies around one of the nodal axes for the M1, while it lies in the anti-nodal area for M2.

Fig. 2 captures the magnitude response of the test resonator with temperature, while the extracted shifts in resonant frequencies are plotted in Figs. 3(a) and 3(c). Linearly fitted frequency shift data from the control resonator is negated from the raw data of the test resonator in order to isolate changes due to the observed phase transition. All the frequency shifts are measured from the nominal frequency of the test resonator measured at the lowest temperature. As the temperature is increased from $290 \mathrm{~K}$ to $320 \mathrm{~K}$ at $1 \mathrm{~K} /$ step, a negative shift in the resonant frequencies for both the modes is observed. This is due to the dependence of elastic moduli of resonator layers on temperature, which is conventionally expressed in the form of the temperature coefficient of frequency (TCF). Post $309 \mathrm{~K}-310 \mathrm{~K}$, a relatively large negative shift is observed for both M1 and M2. The negative trend in the resonant frequency of M1 continues for the next 6-7 K increase in temperature and becomes less steep at approximately $317 \mathrm{~K}-318 \mathrm{~K}$. This observed phase transition temperature width of $309 \mathrm{~K}-318 \mathrm{~K}$ is consistent with the melting
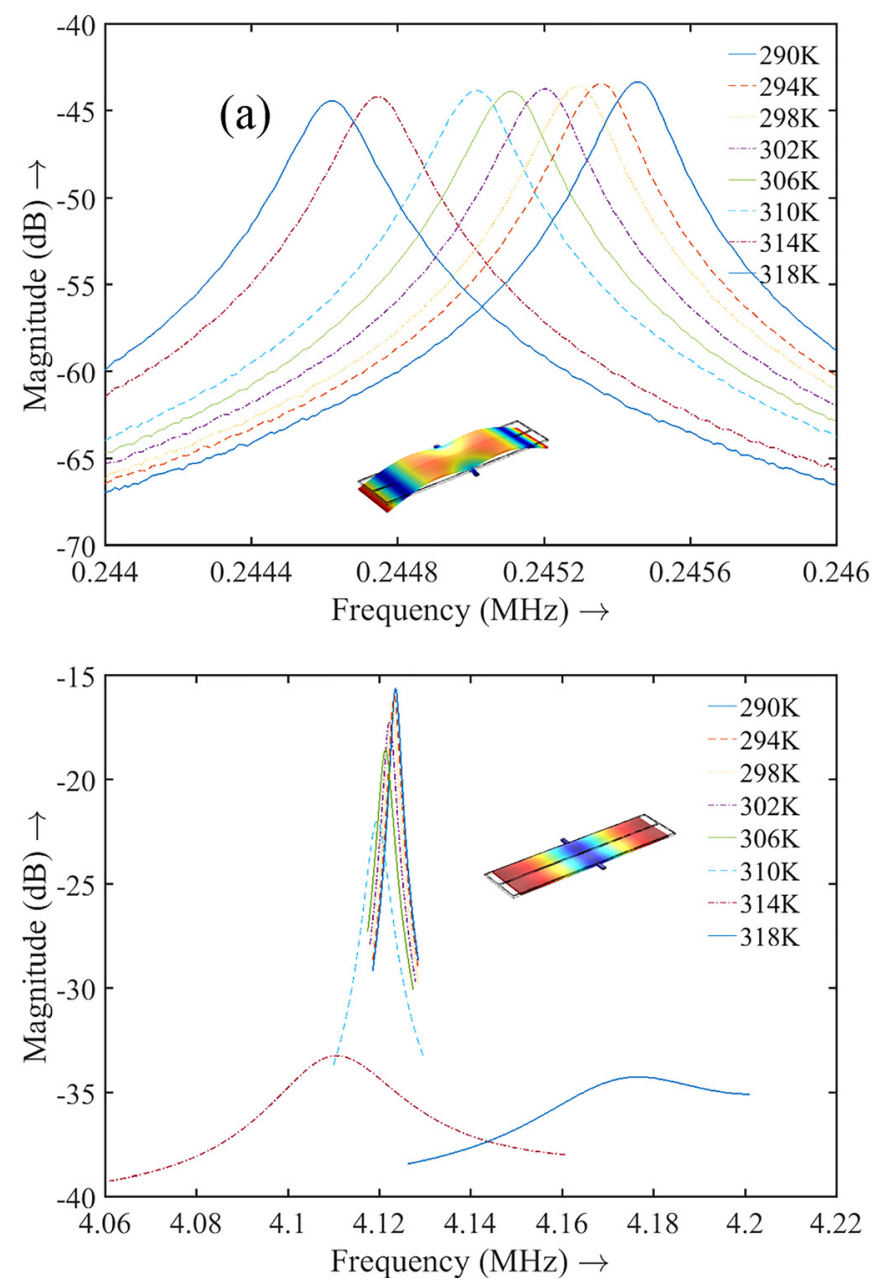

FIG. 2. Magnitude response plots for (a) the flexural mode and for (b) the bulk mode for different temperatures. COMSOL mode shape simulation for both the modes is provided in the inset of the graphs. 

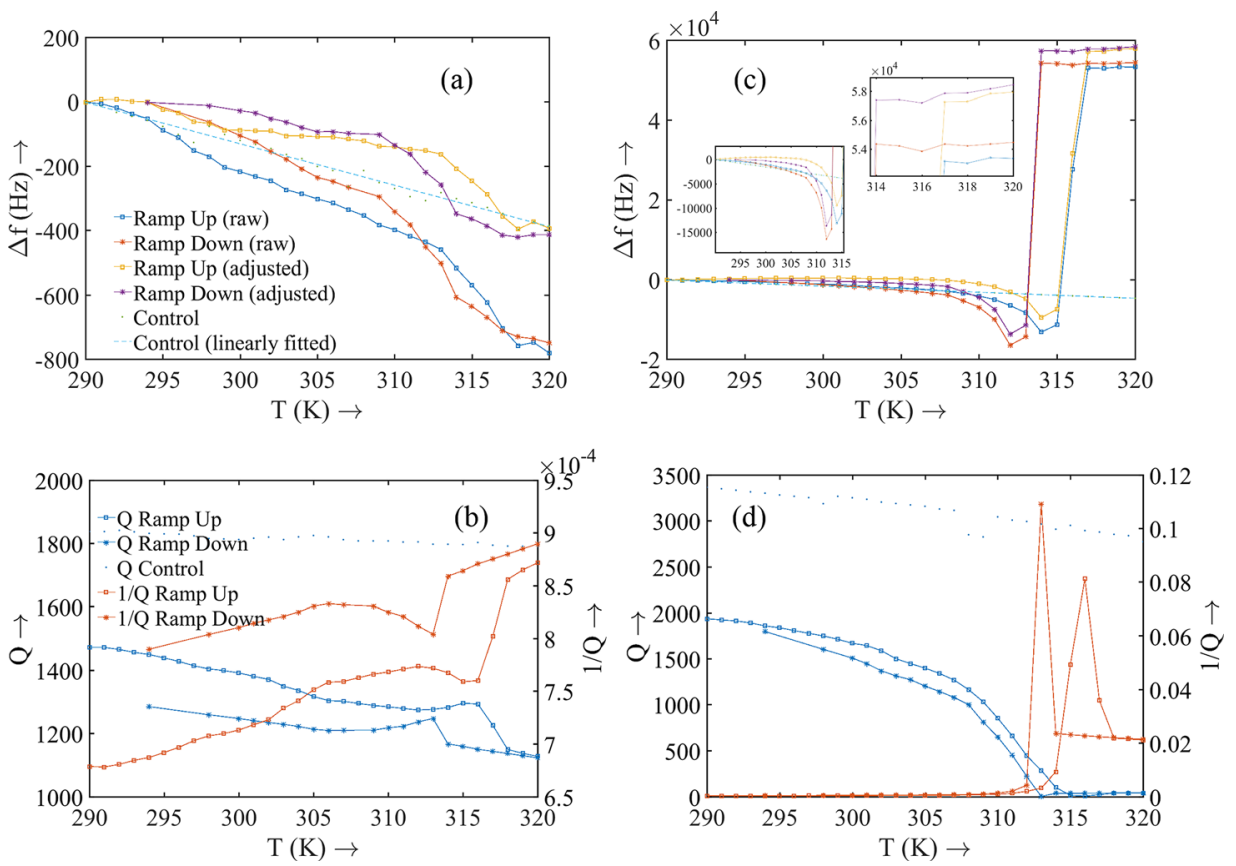

FIG. 3. Frequency shift and $\mathrm{Q}, \mathrm{Q}^{-1}$ vs. temperature plots for the flexural mode (a) and (b) and for the bulk mode (c) and (d) respectively. In (a) and (c), frequency shift values of the test resonator (with the PEG deposit on one of the surfaces) are adjusted by subtracting the frequency shift values from the control resonator (which has a pristine clean surface). In (b) and (d), dissipation factor $(1 / \boldsymbol{Q})$ values for the test resonator are adjusted by subtracting the dissipation factor values (not shown on the plots) obtained from the control resonator. point of PEG 1000 (between $308 \mathrm{~K}$ and $313 \mathrm{~K}$ ) quoted in the product datasheet. ${ }^{23}$ In the case of $\mathrm{M} 2$, a large negative frequency shift is abruptly followed by a large positive shift in the frequency.

Although, it is challenging to address the relationship between the observed resonant behavior to the complex changes associated with the phase transition, qualitative arguments and hypothesis related to the observations are proposed here which remain to be validated in the follow-up studies. Both the modes are expected to acoustically couple with the PEG mass differently due to the inherent difference in the interaction of transverse and longitudinal modal motion with the deposited material. For example, in the case of flexural modes in micro-/nano-cantilevers, the effect of adsorbate stiffness on flexural rigidity has been shown increasingly important as the adsorbate dimensions become comparable to the device thickness. ${ }^{24}$ In the case of bulk modes, changes in resonance frequency due to the deposition of thin-films have been explained by considering both mass as well as bi-axial and shear moduli of the deposited films. ${ }^{25}$ When the PEG mass is solid, it has a finite contribution towards the effective stiffness of the resonator. As the drop starts to melt, this stress related to elastic moduli of the solid material decreases due to the softening of the material resulting in a negative perturbation in stiffness, and hence, a drop in the resonance frequency (from Eq. (1)). A similar negative trend in frequency has been observed in flexural plate wave resonators when the coated polymer transitions from a harder (higher stiffness) physical state to a softer rubbery (less stiff) physical state. ${ }^{22}$ As the PEG mass completely melts, the effect of stiffness becomes negligible (liquid only applies viscous drag which effects the associated liquid mass loading and dissipation). In the case of M2, as the drop completely melts, there is a sudden decrease in the mass loading as only a thin layer of liq$\mathrm{uid}^{26}$ remains in shear contact with the vibrating surface of the resonator. The effective mass loading in such a regime can be obtained by calculating the mass of liquid trapped within the penetration depth $\delta_{\text {pen }}$

$$
\delta_{p e n}=\sqrt{\frac{\eta}{\pi f \rho}}
$$

where $\eta$ is the dynamic viscosity and $\rho$ is the density of the liquid. From Ref. 23 , post phase transition, $\eta / \rho$, or kinematic viscosity of PEG decreases non-linearly with temperature, indicating that as the temperature increases, the penetration depth becomes smaller. This leads to smaller mass loading with the increase in temperature, and, hence, a positive frequency shift. In the case of M1, even in the liquid phase the whole drop remains acoustically coupled to the resonator. While an accurate formulation for such a coupling is required to predict mass loading, it can be safely assumed that due to the nodal location of the drop, any change in mass loading is relatively small.

Quality factors for the resonant peaks are calculated by fitting the individual magnitude responses to a Lorentzian followed by extraction of $3 \mathrm{~dB}$ bandwidth values. In the case of M2 when the peaks are too damped, the phase-slope method $^{27}$ is instead used for the extraction of quality factors which gives a close approximation of the actual $Q$ values. Dissipation factor $(1 / Q)$ values are also obtained for the test and the control resonator. Subtracting the control dissipation factor values from the test resonator values isolates the contribution of the PEG deposit on the damping response of the test resonator. From Figs. 3(b) and 3(d), as the temperature increases, there is a negative shift in the $Q$ values for both M1 and M2. This negative shift resembles characteristic thermo-elastic dissipation (TED) related losses in acoustic resonators. $^{4,28}$ Since, we are more focused on abrupt and large changes in the $\mathrm{Q}$ values which might be caused due to the phase transition, we did not further pursue modelling TED and other loss mechanisms for our resonators. In the case of M2, the rate of quality factor shift increases around $310 \mathrm{~K}$, dropping from $\sim 1200(309 \mathrm{~K})$ to $\sim 10$ to 20 $(314 \mathrm{~K}-315 \mathrm{~K})$. In the case of M1, a noticeable drop in the quality factor is observed after a small bump in the range of of $310 \mathrm{~K}-315 \mathrm{~K}$. When the drop is solid, layers of PEG close 
to the resonator surface are expected to be elastically attached with the resonator surface, causing lower viscoelastic damping. As soon as the melting starts, damping is also expected to increase as more energy is lost within the entrained liquid layers due to shear viscous losses. Dynamic Mechanical Analysis of longer PEGs (4000, 6000, and 20000 ), which has been reported elsewhere, ${ }^{29}$ correlates well with the above observation. As PEG reaches its melting point, slippage between the long molecular chains starts, resulting in a continuous drop in the storage modulus (thereby, decreasing the overall stiffness loading of the resonator) with simultaneous increase in $\tan \delta$ (ratio of loss to storage moduli where $\delta$ is the phase lag between stress and strain; 0 (elastic material) $<\delta<\pi / 2$ (purely viscous)) indicating an increase in dissipation within the material (and hence, a decrease in the overall $\mathrm{Q}$ factor). Flow starts at the melting point leading to a complete drop in the storage modulus. In this regime, the liquid phase dominates the viscoelastic loading of the resonator. Hysteresis is observed in both the modes as well. One possible explanation could be the formation of thermal gradient within the PEG drop. Due to poor thermal conductivity of PEG, different areas within the PEG drop could be at different temperatures. This might result in a thermal lag between the melting and the solidifications cycle. By manipulating the heating and the cooling rates and the mass of the PEG drop, one can establish the extent of contribution of thermal gradient formation on the observed hysteresis. The effect of vibrational frequency and amplitude on hysteresis can also be ascertained by observing lower, intermediate, and higher frequency modes, and by changing the drive amplitudes in the each case. Thermal expansion due to temperature increase and absorbent losses for the heating cycle could also contribute in the observed hysteresis.

In conclusion, micromechanical bulk acoustic wave resonators were found to be sensitive to the phase transition of polyethylene glycol demonstrating a notable degree of changes in the frequency response at different stages of phase change. By addressing different resonant modes, the phase transition of PEG was qualitatively analyzed. A future direction for this work is to establish an analytical acoustic coupling model, which could further help in deriving the mechanical properties of the sample material. Another interesting future direction is to explore the relationship between the heating rate and the phase transition response to establish the accuracy of the method and compare it with other standard techniques. ${ }^{30}$ While the current protocol limits the minimum sample mass which could be deposited, integrating the set-up with a dispensing technique that can precisely and repeatedly dispense the sample material onto the resonator surface would enable further scaling down of the sample mass, while obtaining statistically accurate thermal response curves.

See supplementary material provided for the fabrication process flow and for a detailed discussion on the experimental set-up.

This research was supported by the Cambridge Trusts. The authors would like to thank Dr. Jerome Charmet for the valuable discussions and set-up of the experiments.
${ }^{1}$ A. K. Naik, M. S. Hanay, W. K. Hiebert, X. L. Feng, and M. L. Roukes, "Towards single-molecule nanomechanical mass spectrometry," Nat. Nanotechnol. 4, 445-450 (2009).

${ }^{2}$ A. Prasad, A. T.-H. Lin, V. R. Rao, and A. A. Seshia, "Monitoring sessile droplet evaporation on a micromechanical device," Analyst 139, 5538-5546 (2014).

${ }^{3}$ J. Mertens, E. Finot, T. Thundat, A. Fabre, M.-H. Nadal, V. Eyraud, and E. Bourillot, "Effects of temperature and pressure on microcantilever resonance response," Ultramicroscopy 97, 119-126 (2003).

${ }^{4}$ B. Kim, M. A. Hopcroft, R. N. Candler, C. M. Jha, M. Agarwal, R. Melamud, S. A. Chandorkar, G. Yama, and T. W. Kenny, "Temperature dependence of quality factor in MEMS resonators," J. Microelectromech. Syst. 17, 755-766 (2008).

${ }^{5}$ K. A. Marx, "Quartz crystal microbalance: A useful tool for studying thin polymer films and complex biomolecular systems at the solution-surface interface," Biomacromolecules 4, 1099-1120 (2003).

${ }^{6} \mathrm{H}$. Campanella, Acoustic Wave and Electromechanical Resonators: Concept to Key Applications (Artech House, 2010), available at https://books.google.com/ books?id=ps64Hun $17 \mathrm{wcC} \&$ pgis $=1$.

${ }^{7}$ O. Cakmak, C. Elbuken, E. Ermek, A. Mostafazadeh, I. Baris, B. Erdem Alaca, I. H. Kavakli, and H. Urey, "Microcantilever based disposable viscosity sensor for serum and blood plasma measurements," Methods 63, 225-232 (2013).

${ }^{8}$ J. E. Y. Lee, B. Bahreyni, Y. Zhu, and A. A. Seshia, “A single-crystal-silicon bulk-acoustic-mode microresonator oscillator," IEEE Electron Device Lett. 29, 701-703 (2008).

${ }^{9}$ J. T. M. Beek, P. G. Steeneken, and B. Giesbers, "A 10 MHz piezoresistive MEMS resonator with high Q," in IEEE International Frequency Control Symposium Exposition (2006), pp. 475-480.

${ }^{10}$ A. Hajjam, A. Rahafrooz, J. C. Wilson, and S. Pourkamali, "Thermally actuated MEMS resonant sensors for mass measurement of micro/nanoscale aerosol particles," in 2009 IEEE Sensors (2009), pp. 707-710.

${ }^{11}$ G. Piazza, P. J. Stephanou, and A. P. Pisano, "Piezoelectric aluminum nitride vibrating contour-mode MEMS resonators," J. Microelectromech. Syst. 15, 1406-1418 (2006).

${ }^{12}$ S. Jiang and S. L. Nail, "Effect of process conditions on recovery of protein activity after freezing and freeze-drying," Eur. J. Pharm. Biopharm. 45, 249-257 (1998).

${ }^{13}$ J. L. Keddie, R. A. L. Jones, and R. A. Cory, "Size-dependent depression of the glass transition temperature in polymer films," Europhys. Lett. 27, 59-64 (1994).

${ }^{14}$ A. Pekarovicova, H. Bhide, P. D. Fleming, and J. Pekarovic, "Phasechange inks," J. Coat. Technol. 75, 65-72 (2003).

${ }^{15}$ P. A. Evans, K. Hawkins, R. H. K. Morris, N. Thirumalai, R. Munro, L. Wakeman, M. J. Lawrence, and P. R. Williams, "Gel point and fractal microstructure of incipient blood clots are significant new markers of hemostasis for healthy and anticoagulated blood," Blood 116, 3341-3346 (2010).

${ }^{16} \mathrm{P}$. Gill, T. T. Moghadam, and B. Ranjbar, "Differential scanning calorimetry techniques: applications in biology and nanoscience," J. Biomol. Tech. 21, 167-193 (2010).

${ }^{17}$ Y. Wei, G.-W. Jang, K. F. Hsueh, E. M. Scherr, A. G. MacDiarmid, and A. J. Epstein, "Thermal transitions and mechanical properties of films of chemically prepared polyaniline," Polymer (Guildf) 33, 314-322 (1992).

${ }^{18}$ L. M. Her, R. P. Jefferis, L. A. Gatlin, B. Braxton, and S. L. Nail, "Measurement of glass transition temperatures in freeze concentrated solutions of non-electrolytes by electrical thermal analysis," Pharm. Res. 11, 1023-1029 (1994).

${ }^{19}$ A. Bogdan, M. J. Molina, H. Tenhu, and T. Loerting, "Multiple glass transitions and freezing events of aqueous citric acid," J. Phys. Chem. A 119, 4515-4523 (2015).

${ }^{20}$ S. Ge, Y. Pu, W. Zhang, M. Rafailovich, J. Sokolov, C. Buenviaje, R. Buckmaster, and R. M. Overney, "Shear modulation force microscopy study of near surface glass transition temperatures," Phys. Rev. Lett. 85, 2340-2343 (2000).

${ }^{21} \mathrm{~N}$. Jung, M. Yun, and S. Jeon, "Phase transitions between the rotator phases of paraffin investigated using silicon microcantilevers," J. Chem. Phys. 136, 104903 (2012).

${ }^{22}$ J. W. Grate, S. W. Wenzel, and R. M. White, "Frequency-independent and frequency-dependent polymer transitions observed on flexural plate wave ultrasonic sensors," Anal. Chem. 64, 413-423 (1992).

${ }^{23}$ See http://msdssearch.dow.com/PublishedLiteratureDOWCOM/dh_0889/ 0901b80380889761.pdf?filepath=polyglycols/pdfs/noreg/118-01801.pdf\& fromPage $=$ GetDoc for Technical Data Sheet CARBOWAX Polyethylene Glycol (PEG) 1000 (2011). 
${ }^{24}$ J. Tamayo, D. Ramos, J. Mertens, and M. Calleja, "Effect of the adsorbate stiffness on the resonance response of microcantilever sensors," Appl. Phys. Lett. 89, 224104 (2006).

${ }^{25}$ A. Prasad, J. Charmet, and A. A. Seshia, "Simultaneous interrogation of high-Q modes in a piezoelectric-on-silicon micromechanical resonator," Sens. Actuators, A 238, 207-214 (2016).

${ }^{26}$ K. K. Kanazawa and J. G. Gordon, "Frequency of a quartz microbalance in contact with liquid," Anal. Chem. 57, 1770-1771 (1985).

${ }^{27} \mathrm{~T}$. Satogata, http://toddsatogata.net/2013-USPAS/ResonantDrivenOscillator.pdf for Oscillators, Resonances, and Lorentzians (2013).
${ }^{28}$ C. Tu and J. E.-Y. Lee, "Effects of cryogenic cooling on the quality factor of lamb wave mode aluminium nitride piezoelectric-on-silicon MEMS resonators," Sens. Actuators, A 244, 15-23 (2016).

${ }^{29} \mathrm{See} \quad$ http://www.perkinelmer.com/lab-solutions/resources/docs/APP TgandMeltofPolyethylene.pdf PerkinElmer, Tg and Melting point of a series of Polyethylene Glycols using the material pocket.

${ }^{30}$ R. M. Saeed, J. P. Schlegel, C. Castano, and R. Sawafta, "Uncertainty of thermal characterization of phase change material by differential scanning calorimetry analysis," Int. J. Eng. Res. Technol. 5, 405-412 (2016). 